\title{
EFFECT OF LIGHT CURING PROTOCOL ON THE DEGREE OF CONVERSION OF A LIGHT CURED RESIN CEMENT
}

\author{
Ahmed Ezzat Sabet* and Kamal Khaled Ebeid*
}

\begin{abstract}
Objective: This study was conducted to detect the effect of different modes of light curing on the degree of conversion of a light cured resin cement.

Materials and methods: Three types of different ceramic materials with shade A3 were divided into 3 groups ( $\mathrm{n}=60$ ) (Group 1; resin nano-ceramic material (CERASMART, GC, Tokyo Japan), Group 2; Polymer reinforced ceramic (Vita Enamic, Vita Zahnfabrik, Germany) and Group 3; feldspathic porcelain (Vita MARK II, Vita Zahnfabrik). Each group was then divided into three subgroups ( $n=20)$ according to the light curing mode (subgroup A; low intensity curing, Subgroup B; high intensity curing, and subgroup C; soft start curing). Subgroups were then divided into two divisions according to the curing time (divison I; 10 seconds and divison II; 20 seconds). Degree of conversion of resin cement beneath different kinds of ceramic was tested using FTIR spectroscopy.
\end{abstract}

Results: Three-way ANOVA revealed statistical significance between the three variables. Significant interactions were also observed. The type of ceramic and mode of curing showed significant differences on the degree of conversion of resin cement. Curing time had no significant effect on the degree of conversion of resin cement.

Conclusion: Type of ceramic and curing mode used had a direct effect on the degree of conversion of resin cement.

\section{INTRODUCTION}

Resin cements are used routinely nowadays under all ceramic restorations due to their favourable mechanical properties, superior bond strength, low degree of solubility which all offer further reinforcement to the all ceramic restorations ${ }^{[1]}$. Light-cured (LC) cements are used under thin and translucent restorations where there is adequate light transmission. They provide superior esthetics compared to dual and chemical- cured cements due to the absence of aromatic amines ${ }^{[2]}$. However, when the restoration thickness is above 1.5-2 mm or its opacity inhibits light transmission, the use of dual- cure (DC) resin cements is recommended ${ }^{[3]}$.

The degree of conversion is the ratio between the remaining aliphatic $\mathrm{C}=\mathrm{C}$ double bonds in a cured specimen to the total number of $\mathrm{C}=\mathrm{C}$ bonds in the

* Department of Fixed Prosthodontics, Faculty of Dentistry, Ain Shams University 
uncured sample ${ }^{[4]}$. The longevity of resin bonded all ceramic restorations depends on the sufficient polymerization of the resin cement. Low degrees of conversion are attributed with toxicity from residual monomer, post-operative hypersensitivity, color changes and decreased bond strength increasing the risk of micro-leakage, bacterial ingress and caries ${ }^{[5,6]}$. Monomers elute out of the matrix by the ingress of solvents, causing the expansion of spaces between the polymeric chains leading to the dissolution of the monomers ${ }^{[7]}$.

Many factors affect the degree of conversion and should be taken into consideration as thickness of ceramic restoration, type and shade of ceramic used, composition of resin cement, intensity, time, mode, distance and light curing device used. Light emitting diodes (LED) units or quartz-tungstenhalogen (QTH) can be used to activate these light cured materials. The QTH device has been used for a long time in dentistry, but it shows irradiance reduction over time by the lamp and degradation, due to the bulb overheating ${ }^{[8]}$. LED showed less degradation, blue emission without filter. However, 1st and 2nd generation LED devices have a narrow wavelength spectrum, specific for a camphoroquinone photoinitiator, unlike QTH and 3rd LED generation curing units, which show a wide spectrum ${ }^{[8,9]}$. Different light intensities, curing modes and distance were found to affect the degree of conversion of composites ${ }^{[10,11]}$.

The light curing protocol has a significant effect on the degree of conversion, studies have shown that the distance of light curing unit's tip from the composite affect the degree of conversion significantly due to the decrease in light intensity reaching the monomers ${ }^{[12]}$. Studies have shown higher degrees of polymerization on the upper surfaces near the light than the deeper ones [11]. Different curing modes has been also found significant. Stresses arising from post-gel polymerization shrinkage may produce defects in composite tooth bond, leading to bond failure with associated postoperative sensitivity, microleakage and recurrent caries ${ }^{[13]}$. If the composite-tooth bond is good, it may also cause deformation of the surrounding tooth structure, resulting in microcracks in the cervical enamel that predisposes the tooth to fracture.

One way of minimizing polymerization shrinkage of light-activated composites is to allow flow through controlled polymerization during setting ${ }^{[14]}$. This may be achieved by applying short pulses of light energy, pre-polymerization at low light intensity followed by final cure at high intensity (soft start polymerization) or a combination of both. Studies have shown that these polymerization modes result in smaller marginal gap, increased marginal integrity and improved material properties ${ }^{[15-17]}$. However, only a few studies have reported the effectiveness of cure of these new curing modes ${ }^{[18-21]}$. These studies focused on soft start polymerization, not pulse activation or a combination of both curing modes.

Thus, this study was aimed to detect the effect of different curing modes, and curing times on the degree of conversion of resin cement under different types of ceramics.

\section{MATERIALS AND METHODS}

Three types of different ceramic materials with shade A3 were divided into 3 groups $(n=60)$ (Group 1; resin nano-ceramic material (CERASMART, GC, Tokyo Japan), Group 2; Polymer reinforced ceramic (Vita Enamic, Vita Zahnfabrik, Germany) and Group 3; feldspathic porcelain (Vita MARK II, Vita Zahnfabrik). Each group was then divided into three subgroups $(n=20)$ according to the light curing mode (subgroup A; low intensity curing, Subgroup B; high intensity curing, and subgroup C; soft start curing). Subgroups were then divided into two divisions according to the curing time (divison I; 10 seconds and divison II; 20 seconds). 


\section{Specimen fabrication}

Ceramic plates having dimensions of $14 \times 14$ $\mathrm{mm}$ and $1.0 \mathrm{~mm}$ thickness were produced from ceramic blocks by cutting using a low speed diamond saw (Buehler-Isomet LakeBulff, IL, USA) and then verified by a digital caliper. Specimens were finished and polished using $\mathrm{SiC}$ paper till 1200 grits. Teflon mould was fabricated to ensure a standard thickness of resin cement samples, with an external diameter of $20 \mathrm{~mm}$ and $3 \mathrm{~mm}$ thickness (Fig. 1). An inner dimension $14 \times 14$ square shape was cut with thickness of $1.1 \mathrm{~mm}$ to accommodate the designated thickness of ceramic slice $1.0 \mathrm{~mm}$ and to ensure a $0.1 \mathrm{~mm}$ uniform cement thickness. The ceramic slices were seated on the inner stopper of the mould. The lower opening which is a square of $14 \times 14 \mathrm{~mm}$ used for the placement of the ceramic disc and the resin cement this distance is measured from the outer surface to the inner shoulder.

Translucent light cured resin cement (RelyX Veneer, 3M ESPE, USA) was dispensed from the syringe on the disc, a celluloid strip placed and then the glass slab was applied with finger pressure to ensure complete seating and uniform $0.1 \mathrm{~mm}$ thickness of cement created by the mould. The glass slab was then inverted, and the upper opening was used for curing the resin cement through the ceramic disc), making the mould and ceramic slice underneath. A light curing device (Bluephase, Ivoclar Vivadent, Schaan, Lichtenstein) was used to cure the resin specimens (Fig.2).

The celluloid strip was then removed from the sample and the cement specimen separated from the ceramic slice. The thickness of specimens was verified by a digital caliper. The specimens were kept for twenty-four hours in a dark container to prevent further polymerization and then subjected to analysis.

\section{Degree of conversion measurement}

To obtain the degree of conversion from the given equation, separate values for cured samples as

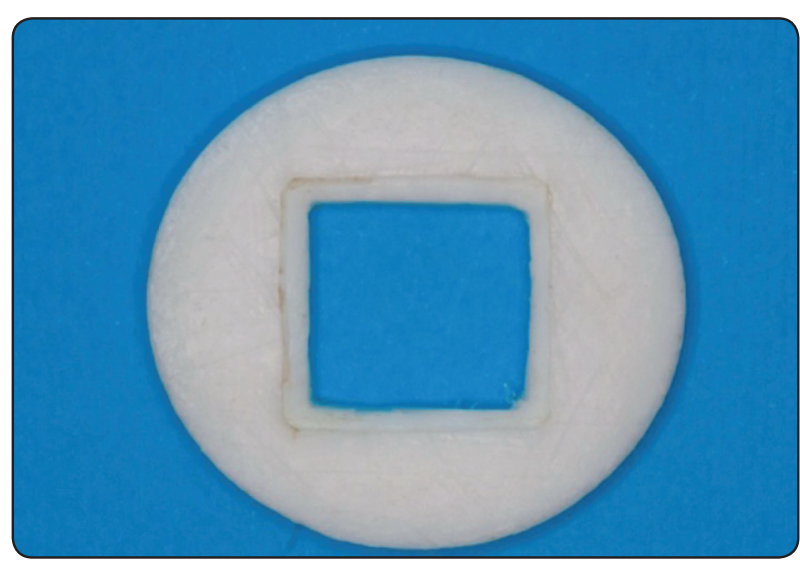

Fig. (1) Teflon mould showing the internal stop

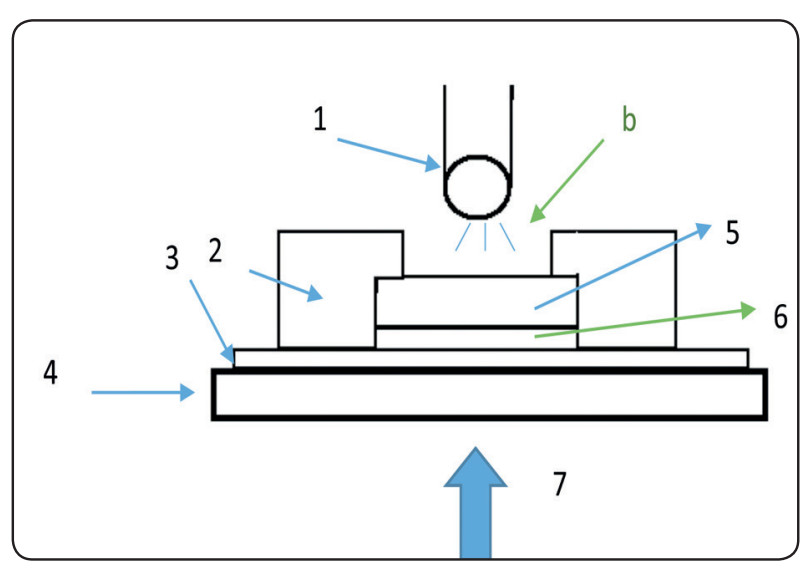

Fig. (2) Diagrammatic representation of the resin/ceramic sample preparation1)Tip of the light curing device 2) Custom made Teflon mould 3) Celluloid strip 4) Glass slab 5) Ceramic disc 6) Resin cement standardized to $0.1 \mathrm{~mm}$ thickness 7) Finger pressure on outer surface of the glass slab

well as raw uncured samples should be calculated. These values were then substituted in the equation and the percentage degree of conversion calculated. Uncured cement specimens were dispensed from the syringe, mixed with potassium bromide powder and compacted to form a disc shaped specimen for FTIR spectroscopy. Each one of cured samples already prepared, was ground into fine powder and mixed with potassium bromide powder. The mixture was compacted to form a disc shaped specimen for the FTIR spectroscopy.

Cured and uncured cement samples were collected after preparation and scanned at a resolution 
of $4 \mathrm{~cm}-1$ and given a blot of wave number from 4000-400 cm-1 against absorbance peak intensities, using OMNIC 5.1c software connected to the FTIR unit (Fourier transform infrared spectroscopy) NICOLET 6700 (Nicolet Instrument Company, USA).

Degree of conversion for each specimen was evaluated using Fourier Transform Infra-Red spectroscopy. In the MIR region, DC was determined by measuring the intensity (or area) decrease of the methacrylate $(\mathrm{C}=\mathrm{C})$ stretch absorption band at the absorption peaks of the aromatic double bonds were recorded at $1608 \mathrm{~cm}-1$ (Abs 1608) and the peak of the aliphatic double bonds $(\mathrm{C}=\mathrm{C})$ were registered at $1637 \mathrm{~cm}-1$ (Abs 1637 as the methacrylate monomer is converted to polymer.

The percentage of unreacted aliphatic $\mathrm{C}=\mathrm{C}$ bonds remaining throughout the polymerization reaction was obtained by the equation:

$$
(\% \mathrm{C}=\mathrm{C})=\frac{[\text { Abs(aliphatic) } / \text { Abs (aromatic) }]_{\text {polymer }}}{[\text { Abs(aliphatic) } / \text { Abs(aromatic) }]_{\text {monomer }}} \times 100
$$

DC was determined by subtracting the residual percentage of aliphatic $\mathrm{C}=\mathrm{C}$ from $100 \%(D C \%=100$ $(\% \mathrm{C}=\mathrm{C})$

Data was then collected, tested for normal distribution then analyzed using three-way ANOVA and tukeys post-hoc tests.

\section{RESULTS}

Three-way ANOVA revealed statistical significance between the three variables (Table 1). Significant interactions were also observed. The type of ceramic and mode of curing showed significant differences on the degree of conversion of resin cement. Curing time had no significant effect on the degree of conversion of resin cement.

For ceramic type, one-way ANOVA revealed statistical significance between the three groups. Tukeys post-hoc test revealed that Vita Mark II group showed the highest degree of conversion of resin cement, followed by Vita Enamic then Cerasmart.

As for mode of curing one-way ANOVA and tukeys post-hoc test revealed no significant differences between the low and high intensity curing modes. However, both showed statistically significant lower degree of conversion when compared to soft start curing mode.

\section{DISCUSSION}

The degree of conversion has a direct influence on the physical, biological and mechanical properties of composite resins. Studies showed that these properties improve by the increase in degree of conversion attained during photo-polymerization ${ }^{[22]}$. Moreover, the increased cure leads to less amount of uncured, potentially leachable monomer making the restorations more biocompatible ${ }^{[23]}$. In addition, functional groups that remain uncured affect the mechanical properties of the resin by acting as a plasticizer $^{[24]}$.

Unreacted monomers are known as residual monomers, the most desirable outcome of any polymerization reaction, is the complete conversion of monomers to polymers. The degree of conversion TABLE (1) Mean and standard deviation of all subgroups

\begin{tabular}{|l|c|c|c|c|c|c|}
\hline \multirow{2}{*}{ Ceramic } & \multicolumn{2}{|c|}{ Vita Mark II } & \multicolumn{2}{c|}{ Vita Enamic } & \multicolumn{3}{c|}{ Cerasmart } \\
\hline & Sec 10 & Sec 20 & Sec 10 & Sec 20 & Sec 10 & Sec 20 \\
\hline Soft & $\pm 3.1^{\mathrm{a}} 59$ & $2.7^{\mathrm{d}} \pm 65$ & $2.7^{\mathrm{c}} \pm 53$ & $3.3 \mathrm{a} \pm 57$ & $2.5^{\mathrm{b}} \pm 49$ & $2.1^{\mathrm{b}} \pm 51$ \\
\hline Low & $1.7^{\mathrm{b}} \pm 51$ & $1.3^{\mathrm{a}} \pm 56$ & $1.3^{\mathrm{b}} \pm 49$ & $2.9^{\mathrm{b}} \pm 50$ & $2.1^{\mathrm{e}} \pm 45$ & $2.2^{\mathrm{e}} \pm 46$ \\
\hline High & $2.2^{\mathrm{c}} \pm 54$ & $1.8^{\mathrm{a}} \pm 57$ & $1.8^{\mathrm{b}} \pm 48$ & $2.1^{\mathrm{b}} \pm 51$ & $1.9^{\mathrm{e}} \pm 43$ & $2.9^{\mathrm{e}} \pm 47$ \\
\hline
\end{tabular}


defines the efficiency of the polymerization process; the higher number of $(\mathrm{c}=\mathrm{c})$ bonds converted into (cc) single bonds affects the polymer network making it more resistant ${ }^{[25]}$.

Several factors affect the degree of polymerization of resin cement as shade, thickness and translucency of the ceramic used, resin cement composition and mode of polymerization. The type of light cure, curing output power, duration and distance also affect the cement polymerization ${ }^{[26]}$. Therefore, ceramic type, curing mode and time were included as variables in this study.

In this study, a light-cured cement was investigated. The use of light cured resin cement has always been an advantage when it comes to cementing laminate veneers. Their color stability; due to the absence of amines, single paste formulation and longer working time, have made it a better choice over dual-cured cements ${ }^{[27]}$. The properties of lightcured cements are influenced by the thickness and composition of the ceramic used. This thickness affects the transmission of light ${ }^{[3]}$ and its quality reaching the underlying cement layer ${ }^{[28,29]}$.

In this study the use of different ceramics showed a significant effect on the degree of conversion of resin cement. Where the polymer infiltrated ceramic Vita Enamic showed higher values than the resin nano ceramic represented by Cerasmart while the feldspathic porcelain represented by the Vita Mark II showed the highest degree of conversion of resin cement. The optical properties of different ceramics depend on the mode of fabrication, chemical structure and the material's microstructure represented by the crystallinity, filler content, chemical nature and whether it has an amorphous phase or not ${ }^{[30]}$. This difference might be attributed to the difference in microstructure and translucency of different types of ceramics tested with the more translucent ceramic Vita Mark II showing more and better light transmission thus leading to more conversion of the resin cement ${ }^{[31]}$.
As for the curing mode there was no difference between the low and high curing modes. This might be due to the fact that the low curing mode was not enough to obtain enough conversion of the resin cement while the high curing mode only converted monomer on the surface while monomer that was deep was not converted enough ${ }^{[32]}$. The soft start curing mode showed the highest degree of conversion of resin cement and this might be due that soft start curing resulted in eventual curing of the bulk of the cement followed by more conversion as the intensity of light increased.

For curing time there was no effect on the degree of conversion of resin cement showing that 10 seconds curing in all modes was enough to convert monomers in resin cement and further curing had no effect.

\section{CONCLUSION}

Within the limitation of this study we can concluded that:

1) The type of ceramic has a direct effect on the degree of conversion of the resin cement beneath.

2) Mode of curing of resin cement has a direct effect on its degree of conversion.

\section{REFERENCES}

1. Hill E.E. and Lott J. A clinically focused discussion of luting materials. Aust Dent J 2011; 56 Suppl 1:67-76.

2. Souza-Junior E.J., Prieto L.T., Soares G.P., Dias C.T., Aguiar F.H., and Paulillo L.A. The effect of curing light and chemical catalyst on the degree of conversion of two dual cured resin luting cements. Lasers Med Sci 2012; 27:145-51.

3. Lohbauer U., Pelka M., Belli R., Schmitt J., Mocker E., Jandt K.D., and Muller F.A. Degree of conversion of luting resins around ceramic inlays in natural deep cavities: a micro-Raman spectroscopy analysis. Oper Dent 2010; 35:579-86.

4. Moraes L.G., Rocha R.S., Menegazzo L.M., de Araujo E.B., Yukimito K., and Moraes J.C. Infrared spectroscopy: a tool for determination of the degree of conversion in dental composites. J Appl Oral Sci 2008; 16:145-9. 
5. Janda R., Roulet J.F., Kaminsky M., Steffin G., and Latta M. Color stability of resin matrix restorative materials as a function of the method of light activation. Eur J Oral Sci 2004; 112:280-5.

6. Goldberg M. In vitro and in vivo studies on the toxicity of dental resin components: a review. Clin Oral Investig 2008; 12:1-8.

7. Polydorou O., Huberty C., Wolkewitz M., Bolek R., Hellwig E., and Kummerer K. The effect of storage medium on the elution of monomers from composite materials. $\mathrm{J}$ Biomed Mater Res B Appl Biomater 2012; 100:68-74.

8. Faria-e-Silva A.L., Lima A.F., Moraes R.R., Piva E., and Martins L.R. Degree of conversion of etch-and-rinse and self-etch adhesives light-cured using QTH or LED. Oper Dent 2010; 35:649-54.

9. Asmussen E. and Peutzfeldt A. Polymer structure of a light-cured resin composite in relation to distance from the surface. Eur J Oral Sci 2003; 111:277-9.

10. Silikas N., Eliades G., and Watts D.C. Light intensity effects on resin-composite degree of conversion and shrinkage strain. Dent Mater 2000; 16:292-6.

11. Catelan A., Mainardi Mdo C., Soares G.P., de Lima A.F., Ambrosano G.M., Lima D.A., Marchi G.M., and Aguiar F.H. Effect of light curing protocol on degree of conversion of composites. Acta Odontol Scand 2014; 72:898-902.

12. Stansbury J.W. Dimethacrylate network formation and polymer property evolution as determined by the selection of monomers and curing conditions. Dent Mater 2012; 28:13-22.

13. Eick J.D. and Welch F.H. Dentin adhesives--do they protect the dentin from acid etching? Quintessence Int 1986; 17:533-44.

14. Sheth J.J., Fuller J.L., and Jensen M.E. Cuspal deformation and fracture resistance of teeth with dentin adhesives and composites. J Prosthet Dent 1988; 60:560-9.

15. Uno S. and Asmussen E. Marginal adaptation of a restorative resin polymerized at reduced rate. Scand J Dent Res 1991; 99:440-4.

16. Mehl A., Hickel R., and Kunzelmann K.H. Physical properties and gap formation of light-cured composites with and without 'softstart-polymerization'. J Dent 1997; 25:321-30.
17. Kanca J., 3rd and Suh B.I. Pulse activation: reducing resinbased composite contraction stresses at the enamel cavosurface margins. Am J Dent 1999; 12:107-12.

18. Yap A.U., Ng S.C., and Siow K.S. Soft-start polymerization: influence on effectiveness of cure and post-gel shrinkage. Oper Dent 2001; 26:260-6.

19. Bouschlicher M.R., Rueggeberg F.A., and Wilson B.M. Correlation of bottom-to-top surface microhardness and conversion ratios for a variety of resin composite compositions. Oper Dent 2004; 29:698-704.

20. Bouschlicher M.R. and Rueggeberg F.A. Effect of ramped light intensity on polymerization force and conversion in a photoactivated composite. J Esthet Dent 2000; 12:328-39.

21. Bouschlicher M.R., Rueggeberg F.A., and Boyer D.B. Effect of stepped light intensity on polymerization force and conversion in a photoactivated composite. J Esthet Dent 2000; 12:23-32.

22. Lovell L.G., Lu H., Elliott J.E., Stansbury J.W., and Bowman C.N. The effect of cure rate on the mechanical properties of dental resins. Dental Materials 2001; 17:504-511.

23. Yap A.U., Soh M.S., Han T.T., and Siow K.S. Influence of curing lights and modes on cross-link density of dental composites. Oper Dent 2004; 29:410-5.

24. Asmussen E. and Peutzfeldt A. Influence of pulse-delay curing on softening of polymer structures. J Dent Res 2001; 80:1570-3.

25. Yoon T.H., Lee Y.K., Lim B.S., and Kim C.W. Degree of polymerization of resin composites by different light sources. Journal of oral rehabilitation 2002; 29:1165-1173.

26. Heffernan M.J., Aquilino S.A., Diaz-Arnold A.M., Haselton D.R., Stanford C.M., and Vargas M.A. Relative translucency of six all-ceramic systems. Part I: core materials. The Journal of prosthetic dentistry 2002; 88:4-9.

27. Souza-Junior E.J., Prieto L.T., Soares G.P., dos Santos Dias C.T., Aguiar F.H.B., and Paulillo L.A.M.S. The effect of curing light and chemical catalyst on the degree of conversion of two dual cured resin luting cements. Lasers in Medical Science 2012; 27:145-151.

28. Peixoto R.T., Paulinelli V.M.F., Sander H.H., Lanza M.D., Cury L.A., and Poletto L.T.A. Light transmission through porcelain. Dental Materials 2007; 23:1363-1368. 
29. Kilinc E., Antonson S.A., Hardigan P.C., and Kesercioglu A. The effect of ceramic restoration shade and thickness on the polymerization of light- and dual-cure resin cements. Oper Dent 2011; 36:661-9.

30. Subasi M.G., Alp G., Johnston W.M., and Yilmaz B. Effect of thickness on optical properties of monolithic CADCAM ceramics. J Dent 2018.
31. Lee S.M. and Choi Y.S. Effect of ceramic material and resin cement systems on the color stability of laminate veneers after accelerated aging. J Prosthet Dent 2018.

32. Agarwal P.M., Taneja S., and Kumar M. To evaluate and compare the effect of different light-curing modes and different liners on cuspal deflection in premolar teeth restored with bulk filled or incrementally filled composite measured at different time intervals. J Conserv Dent 2017; 20:317-321. 